Meta

Journal des traducteurs

Translators' Journal

\title{
De la coimpression à la réécriture : traduire la fiction anglo-canadienne en coédition
}

\section{Solange Beaulieu et Hélène Buzelin}

Volume 61, numéro 3, décembre 2016

URI : https://id.erudit.org/iderudit/1039217ar

DOI : https://doi.org/10.7202/1039217ar

Aller au sommaire du numéro

\section{Éditeur(s)}

Les Presses de l’Université de Montréal

\section{ISSN}

0026-0452 (imprimé)

1492-1421 (numérique)

Découvrir la revue

\section{Citer cet article}

Beaulieu, S. \& Buzelin, H. (2016). De la coimpression à la réécriture : traduire la fiction anglo-canadienne en coédition. Meta, 61(3), 511-533.

https://doi.org/10.7202/1039217ar
Résumé de l'article

Le présent article porte sur la coédition internationale d'ouvrages de fiction. Il s'intéresse en particulier aux traductions de romans anglo-canadiens récents ayant fait l'objet d'une coédition France-Québec et dans laquelle la maison québécoise agissait à titre d'éditeur de tête. Selon une approche sociotraductologique et à partir de quatre études de cas engageant des éditeurs et traducteurs distincts, les auteures mettent en relief la diversité des pratiques de coédition, les tensions qui les accompagnent et les points de recoupement entre ces différents cas. Elles interrogent aussi les liens, décalages et contradictions entre les discours et les pratiques, et montrent à quel point les pratiques et les perceptions des agents engagés dans ces partenariats sont en partie dépendantes de la position que ceux-ci occupent dans leur champ. 


\title{
De la coimpression à la réécriture: traduire la fiction anglo-canadienne en coédition
}

\author{
SOLANGE BEAULIEU* \\ Montréal, Canada \\ beaulieu.solange@gmail.com \\ HÉLÈNE BUZELIN \\ Université de Montréal, Montréal, Canada \\ helene.buzelin@umontreal.ca
}

\begin{abstract}
RÉSUMÉ
Le présent article porte sur la coédition internationale d'ouvrages de fiction. II s'intéresse en particulier aux traductions de romans anglo-canadiens récents ayant fait l'objet d'une coédition France-Québec et dans laquelle la maison québécoise agissait à titre d'éditeur de tête. Selon une approche sociotraductologique et à partir de quatre études de cas engageant des éditeurs et traducteurs distincts, les auteures mettent en relief la diversité des pratiques de coédition, les tensions qui les accompagnent et les points de recoupement entre ces différents cas. Elles interrogent aussi les liens, décalages et contradictions entre les discours et les pratiques, et montrent à quel point les pratiques et les perceptions des agents engagés dans ces partenariats sont en partie dépendantes de la position que ceux-ci occupent dans leur champ.
\end{abstract}

\begin{abstract}
The current study explores the international copublishing of literary books, in particular that of recent Canadian English novels translated into French by French Canadian publishers and copublished in France. Following a socio-traductological approach, and based on four case studies involving a range of publishers as well as translators, the research highlights the diversity in translation practices while identifying areas of overlap. The article also tackles the following questions: the potential impact of the copublishing model on the linguistic norm adopted by Quebec translators and publishers; how copublishing shapes and is at once informed by the balance of power between the two editorial fields; to what extent perceptions about copublishing experiences by the involved parties are influenced by their position in the editorial field.
\end{abstract}

\section{MOTS CLÉS/KEYWORDS}

coédition internationale, traduction, norme linguistique, littérature, processus éditorial international co-publishing, translation, linguistic norm, literature, editorial process

Dans la foulée de l'adoption de la Loi sur les langues officielles en 1969, le Conseil des arts du Canada (CAC) a mis sur pied un programme de subventions dans le but d'encourager la traduction du canon littéraire national dans les deux langues officielles, le français et l'anglais. Ce programme, inauguré en 1973, allait aussi favoriser, indirectement, le rayonnement de ces littératures à l'échelle internationale, à tout le moins dans le reste de la francophonie (notamment en France) pour la littérature anglo-canadienne et dans d'autres régions du monde anglophone pour les titres d'expression française ${ }^{1}$. L'activité de traduction littéraire au Canada s'est intensifiée 
dans les décennies suivantes, bien que le nombre d'ouvrages subventionnés soit resté relativement stable jusqu'en 2009-2010². Au Québec, les années 1990, décennie pendant laquelle la fiction anglo-canadienne a connu une vague de popularité sur la scène mondiale, ont marqué le début d'une concurrence entre les éditeurs québécois en ce qui concerne l'achat de droits de traduction de titres anglo-canadiens, concurrence qui s'est intensifiée alors que de nouvelles maisons d'édition littéraires voyaient le jour au début des années 2000. C’est dans ce contexte que les éditeurs québécois qui, depuis les années 1980, coéditaient déjà avec leurs homologues français des titres signés par des auteurs québécois (Vaugeois 2002) ont développé des ententes portant aussi sur des ouvrages publiés en traduction. De façon générale, la coédition comporte deux attraits, l'un d'ordre symbolique (ou marketing), l'autre financier. D'une part, elle permet à l'éditeur de tête d'accroître le rayonnement de ses titres à l'étranger en misant sur la notoriété du coéditeur et la solidité de son réseau de distribution sur son marché tout en offrant au coéditeur l'opportunité d'enrichir son catalogue; d'autre part, elle représente (pour l'éditeur de tête comme le coéditeur) une façon de partager certains coûts de production, dans ce cas précis, les dépenses liées à la mise au point de la traduction. Ces coûts étant généralement pris en charge par le CAC lorsque l'auteur et le traducteur sont résidents ou citoyens canadiens, une logique s'est vite - quoiqu'informellement - instaurée entre les éditeurs de France et du Québec: la traduction des ouvrages admissibles au programme du CAC serait réalisée au Québec; celle des autres ouvrages serait plutôt préparée en France ${ }^{3}$.

L'existence et les enjeux de ces partenariats nous sont apparus lors d'une étude de terrain conduite aux éditions du Boréal (Buzelin 2006). Cette étude visait à documenter la genèse d'une traduction littéraire, depuis l'acquisition des droits jusqu'au lancement du livre, et ce, afin de mettre en relief la dimension collaborative du processus et la façon dont l'interaction entre les acteurs y étant investis façonnait le «produit fini». Dans cette étude, qui portait sur un projet de traduction littéraire «classique» pour le Québec ${ }^{4}$, un des acteurs semblait exercer une influence particulière: le coéditeur français. Longtemps invisible et absent, ce coéditeur semblait pourtant tirer indirectement les ficelles. Dès les premières phases du processus, il était un fréquent sujet de conversation entre les professionnels québécois, chacun travaillant à sa façon au «recrutement» de ce partenaire: tandis que la responsable de la vente de droits et le PDG vantaient les mérites du titre anglo-canadien qu'ils avaient acquis et la compétence de leurs traducteurs auprès de collègues français, les traducteurs travaillaient à produire un texte dans une variété de français qu'ils baptisèrent le Mid-Atlantic French. Plus idéalisée que réelle, cette variété renvoyait en fait à un processus de polissage du texte afin d'en éliminer les québécismes, à l'égard desquels les lecteurs français sont chatouilleux, et les anglicismes, qui irritent particulièrement les lecteurs québécois. Cette traduction fut finalement acquise par un coéditeur qui, après relecture de la traduction, proposa tout de même quelques changements que les traducteurs acceptèrent. Si l'histoire de cette traduction (Buzelin 2006; 2007) a fait émerger une dimension et des enjeux jusque-là peu documentés en traductologie, soit l'influence des partenariats internationaux sur les pratiques de traduction et l'ensemble du processus, elle soulève de nouvelles questions: la traduction en Mid-Atlantic French est-elle une pratique courante au Québec? Autrement dit, les traductions de titres ayant un potentiel international sont-elles formatées en vue de séduire d'éventuels coéditeurs et, à travers eux, le public européen? À quel point ces 
coéditeurs peuvent-ils modifier la traduction qu'ils acquièrent et qui a le dernier mot sur les modifications demandées? En somme, quel impact ces partenariats ont-ils sur les choix des traducteurs? Comment sont-ils perçus? Comment sont-ils vécus? Et peut-on dégager des tendances dans les modalités que prennent ces ententes?

Le présent article tente de répondre à ces questions. Il commence par un survol de la documentation sur la coédition internationale (Section 1). Nous présentons ensuite la méthodologie de recherche et résumons les faits saillants relatifs à chacun des quatre cas étudiés (Section 2). Les deux sections suivantes sont consacrées à l'analyse des stratégies adoptées par les traducteurs et aux changements apportés à leurs textes au cours du processus de coédition (Section 3), puis à celle des discours et perceptions des agents engagés dans ces projets de coédition (Section 4). Cette analyse débouche sur une discussion dans laquelle nous mettons en relief les points de recoupement et les éventuelles contradictions entre les discours et les pratiques (Section 5). À ce stade, nous explorons également à quel point la position des acteurs dans leur champ d'activité peut influencer la façon dont ils pratiquent la coédition et se la représentent.

\section{L'édition internationale, la coédition et la traductologie}

Les pratiques de coédition et de coproduction intéressent depuis longtemps les spécialistes de l'imprimé. En 1991, Philippe Schuwer consacrait un traité à ce sujet. Il y définit la coédition internationale comme « un accord pour la traduction-adaptation d'ouvrages généralement illustrés, conçus par un éditeur, détenteur du copyright, qui en cède à un ou à plusieurs confrères étrangers les droits d'édition" (Schuwer 1991: 16). Il distingue clairement cette pratique de la coproduction, laquelle désigne «l'association d'éditeurs pour concevoir, financer, réaliser, et souvent imprimer, un ou plusieurs ouvrages, généralement en diverses langues étrangères, dont ils détiennent ensemble le copyright» (Schuwer 1991: 16). En somme, la coproduction serait un partenariat noué en amont d'un projet éditorial et constituant un préalable à la réalisation de celui-ci, contrairement à la coédition, pratique beaucoup plus courante et moins contraignante reposant minimalement sur une cession de droits.

Sans contester cette distinction entre coédition et coproduction ni les définitions en elles-mêmes, nous avons souligné sur la base d'une étude comparative de sources françaises, québécoises et anglo-saxonnes (Buzelin 2009) à quel point les acceptions de la coédition et les discours entourant ces pratiques variaient largement d'une langue et d'un contexte culturel à l'autre. Dans les sources françaises, par exemple, la coédition est généralement associée à l'édition internationale de livres illustrés et à la pratique de coimpression. Au Québec en revanche, alors que la coédition de livres illustrés existe depuis longtemps, les discours sont plutôt axés sur les ententes relatives à l'édition littéraire et universitaire. Même si elle concerne bien souvent des ouvrages anglo-canadiens ou étrangers publiés en traduction française, la coédition avec des partenaires français y est perçue comme une façon efficace de pénétrer le marché français et d'y faire connaître les auteurs québécois (Filion 1991; Vaugeois 2002; Baju 2004). Cette idée est d'ailleurs réitérée par une éditrice rencontrée dans le cadre de notre étude:

Nous avons recours à la coédition pour permettre à un livre, à un auteur, d'avoir un rayonnement plus large. Il y a un marché en France, et ce marché, je n’y ai pas accès. 
J’y ai accès par la librairie du Québec, mais c'est par la bande, là. S'implanter sur ce marché-là ce serait une fortune, c'est un gouffre. Donc la coédition pour moi, c'est trouver un partenaire avec qui on a des points communs et céder un territoire. (Raoult 2013)

Enfin, les sources d'expression anglaise laissent entrevoir une acception beaucoup plus large de la coédition qui devient quasi synonyme d'une cession de droits et de fichiers, en vue de favoriser la diffusion internationale de titres. Ces écarts notionnels montrent à quel point, si les pratiques éditoriales s'internationalisent, les discours sur ces pratiques demeurent ancrés et influencés par des préoccupations et enjeux très nationaux. L'ensemble de ces discours nous invite à nous représenter la coédition comme un continuum de pratiques très diversifiées allant de la coimpression (acception restreinte correspondant à la forme la plus poussée de coédition) à la «simple» vente de droits avec partage de fichiers (acception large). En ce qui concerne la coédition unilingue de traductions par des éditeurs d'expression française - objet qui nous occupe ici -, l'entente suppose, minimalement, un partage des territoires de la francophonie ainsi que l'acquisition, par le coéditeur, de la traduction réalisée par l'éditeur de tête. Notons que ce partage des territoires peut être tantôt imposé aux deux éditeurs francophones par le détenteur du copyright (qui refuserait de céder les droits mondiaux pour le français à un seul éditeur) ou bien se produire en aval (lorsque l'un des deux éditeurs francophones décide de céder à son homologue une partie des droits de traduction qu'il a acquis).

Outre les définitions et descriptions de ces partenariats, les travaux émanant des spécialistes de l'édition abordent souvent la question de l'impact de la coédition (ou de la coproduction) sur le contenu des livres, sur leur qualité et sur la diversité culturelle. À ce titre, Fouché et al. (voir 2002: 559) résument bien la double facette de la coédition. D'une part, celle-ci encourage la création de titres destinés à un public international aussi large que possible et peut donc contribuer, par là même, à un certain nivellement, voire à une forme d'aseptisation, des contenus; de l'autre, les économies d'échelle qu'elle permet de réaliser facilitent aussi la réalisation de projets ambitieux que des éditeurs n'oseraient peut-être pas entreprendre en solo. Les auteurs soulignent enfin que les coéditions favorisent la circulation des savoirs au-delà des frontières nationales et que ces projets, en mobilisant les meilleures ressources possible à l'échelle internationale, peuvent rehausser la qualité esthétique des livres. Quelques études de cas viennent alimenter la synthèse de Fouché et al. (2002). Par exemple, à partir d'une analyse portant sur un corpus de livres pratiques, Christian Robin (2006) montre que dans ce secteur éditorial, les partenariats de coédition et de coproduction sont devenus la norme et que ces développements ont eu, somme toute, un impact plutôt positif sur la qualité éditoriale des ouvrages. Par ailleurs, l'étude de Hervé Serry (2009) sur l'échec du projet de coédition européenne Faire l'Europe, projet qui portait sur des livres d'histoire, révèle aussi les limites de ces partenariats, soit la difficulté de s'entendre sur les contenus, notamment lorsque ceux-ci touchent des domaines aussi sensibles que l'historiographie. Par ailleurs, les spécialistes de l'imprimé se sont surtout penchés sur les enjeux de la coédition internationale multilingue. En ce qui concerne la coédition unilingue, le nombre de publications est beaucoup plus bas. La seule étude approfondie semble être celle de Sarah Baju (2004) sur le partenariat entre les éditions Actes Sud créées en 1978 à Arles et la maison Leméac fondée à Montréal en 1957. Baju rapporte que le partenariat s'est 
noué en 1989, au moment où ces deux maisons se dotaient de leur propre structure de diffusion. Il s'agit d'une entente fondée sur un principe de réciprocité, chaque maison sélectionnant des titres du catalogue du partenaire, titres qu'elle s'engage à publier et à diffuser sous sa marque sur son territoire. Les points de recoupement entre les lignes éditoriales des deux maisons sont donc essentiels. Chez Leméac, Actes Sud a puisé en priorité des œuvres de théâtre, des romans québécois et, dans une proportion beaucoup plus minime, des œuvres d'expression étrangère. En contrepartie, ce partenariat a surtout permis à Leméac d'ouvrir son catalogue aux auteurs étrangers non francophones découverts par Actes Sud (voir Baju 2004: 101-109). Baju note également que, dès leur création, les éditions Actes Sud se sont distinguées symboliquement par la singularité de leur format $10 \times 19$, mais aussi celle de leurs couvertures et du type de papier utilisé (voir 2004: 51). Or, il est intéressant de constater que «les éditeurs de Leméac publient volontairement les ouvrages qu'ils coéditent de façon à ce qu'ils ressemblent à des livres d'Actes Sud. En effet, les formats, les couvertures, le papier, la typographie, tous les éléments qui font le livre Actes Sud sont conservés par Leméac» (Baju 2004: 110). À l'inverse, Actes Sud conserve sa charte graphique, si particulière, pour les livres de Leméac qu'elle choisit de coéditer. Baju conclut son étude sur la mention suivante:

En l'espace de quatorze ans [1989-2003], les deux éditeurs ont publié ensemble cent trente-quatre titres, soit une moyenne de dix par an environ. Lorsque l'on compare ces chiffres avec les quatre cent cinquante titres qu'Actes Sud publie en moyenne chaque année, cela semble dérisoire. Or, pour Leméac, dont la production annuelle tourne autour de quarante titres par an, ces coéditions représentent près d'un quart de son programme éditorial. (Baju 2004: 116)

En 2013, le nombre total de titres coédités entre ces deux maisons, tous genres confondus, était passé à cent soixante-dix.

Du côté de la traductologie, les mentions et analyses de la coédition demeurent peu nombreuses. L'étude de Dollerup et Orel-Kos (2001) sur la coimpression internationale dans le domaine de la littérature jeunesse est l'une des premières à documenter l'impact de ce type de partenariat sur les pratiques et sur la représentation $\mathrm{du}$ processus de traduction. Les auteurs estiment que les coimpressions sont souvent perçues avec condescendance, ce qui explique en partie le fait qu'elles n'aient guère attiré l'attention des traductologues. Ils expliquent que la coédition de livres pour enfants et de beaux livres est généralement le fait d'un éditeur supranational qui négocie la distribution du livre dans des marchés nationaux. Cette pratique permet la distribution de livres illustrés dans de petits pays et de "petites langues» qui n'y auraient pas accès autrement. La traductrice Josephine Bacon (2005) met quant à elle en relief les limites et ratés de la coédition dans le domaine des livres pratiques, en montrant que ces livres - de recettes, de jardinage, de bricolage... - ne franchissent pas aisément les barrières linguistiques et culturelles. Ses exemples montrent que les éditeurs ne sont pas toujours conscients de ces barrières culturelles et qu'ils auraient donc intérêt à confier à des traducteurs ou à des consultants la tâche d'évaluer le travail d'adaptation nécessaire avant d'acquérir les droits de traduction. À la suite de l'étude de cas conduite aux éditions du Boréal mentionnée en introduction, nous avons répertorié différentes formes de coéditions internationales réalisées par les éditeurs du Québec dans des genres autres que celui de la fiction, et ce, afin de mettre en relief les conditions de possibilité et les limites de ces ententes. Entre autres, ces 
partenariats supposent une certaine forme de diversité et de concurrence, aussi imparfaite soit-elle, entre les maisons d'édition. À contrario, ils deviennent plus rares quand le paysage éditorial se concentre autour de quelques conglomérats et oligopoles $^{5}$. Ces ententes peuvent prendre de multiples formes intégrant, à divers degrés, le dialogue (entre les partenaires), la mise en commun (de ressources et expertises) et une division des tâches (Buzelin 2007). En ce qui concerne la traduction en tant que telle, ces ententes complexifient le processus en multipliant le nombre de participants et de paramètres à considérer. Ainsi dans le cas d'une coimpression, le traducteur devra respecter la composition typographique et l'iconographie conçues par l'éditeur de tête; dans d'autres cas, il devra souvent respecter des délais fixés avec l'éditeur de tête et traduire dans une variété de langues «internationales», en MidAtlantlic French, par exemple, s'il s'agit d'une coédition avec la France. Nous avions alors conclu (Buzelin 2007) que si la coédition est une pratique devenue courante, non seulement dans l'édition de livres illustrés, mais également dans l'édition littéraire et générale, elle pose aussi des défis pour la traductologie, à au moins deux titres. Tout d'abord, l'étude de ces ententes suppose une approche de terrain et un accès au processus éditorial de la part des chercheurs. Par ailleurs, le caractère ponctuel et flexible de ces partenariats - qui se tissent le plus souvent autour d'un titre ou d'une collection et participent d'une «économie de l'instant» (Forget 2004) ${ }^{6}$ - rend toute tentative de généralisation hasardeuse ${ }^{7}$.

C'est dans cet esprit que nous avons entrepris de prolonger le travail amorcé en 2006. Présupposant que les pratiques de coédition sont diversifiées, la présente contribution propose de nouvelles études de cas réalisées auprès de quatre autres maisons d'édition et traducteurs littéraires ${ }^{8}$. Elle porte exclusivement sur des traductions de romans anglo-canadiens préparées pour des éditeurs du Québec et coéditées en France. Partant du constat que l'édition littéraire au Québec constitue un champ, au sens défini par Bourdieu (1991), l'article interroge également en quoi, au-delà d'une diversité de surface, les pratiques et perceptions de la coédition par les professionnels interviewés dépendent en partie de la place que ceux-ci occupent dans leur champ d'activité et plus généralement dans la sphère de production culturelle.

\section{Notes méthodologiques}

\subsection{La sélection des cas}

Notre problématique de recherche suppose une collecte de données auprès de plusieurs maisons d'édition littéraires du Québec et leurs traducteurs. Conduire un sondage nous semblait peu approprié en raison du caractère délicat du sujet traité - la coédition touchant la question des rapports de force et de pouvoir au sein du champ littéraire. Nous avons donc plutôt choisi de conduire de nouvelles études de cas, en les sélectionnant de façon à favoriser la diversité (de titres, de maisons et de traducteurs). Nous avons privilégié des œuvres de fiction récentes signées par des auteurs anglo-canadiens, traduites en français au Québec et coéditées par un éditeur français entre 2003 et 2012. Comme il n'existe aucune base de données recoupant tous les paramètres énoncés ci-dessus, nous avons consulté plusieurs sources, dont chacune fournit des indices différents, afin de dégager une liste générale de titres coédités. De là, nous avons arrêté notre choix en procédant par élimination. 
L'index Translationum de l'UNESCO, qui recense les livres publiés dans les pays membres de l'ONU, permet de suivre l'évolution de la traduction de livres au Canada (voir Tableau 2, en annexe). Jusqu'en 1991, le nombre de traductions restait inférieur à 1000 par année. De 1992 jusqu'à 2008, le nombre de livres traduits n'a cessé de croître jusqu'à atteindre 1606 en 2008, puis à chuter à 323 en 2009. Sur les 1606 traductions de 2008, 1245 avaient pour langue cible le français, 298 l'anglais et 63 traductions concernaient d'autres langues. Au Québec, l’organisme Bibliothèque et Archives nationales du Québec (BANQ) publie chaque année des Statistiques de l'édition sur les ouvrages ayant reçu le dépôt légal (voir les Tableaux 1, 3 et 4 en annexe). Ces statistiques recouvrent tous les genres: documentaires, beaux livres et fiction, en plus de manuels pratiques, de livres pour enfants et d'ouvrages de référence, entre autres. Sur la période étudiée, plus de $85 \%$ des ouvrages déposés à BANQ sont publiés en français, le reste est majoritairement publié en anglais. La catégorie «Langues et littérature» représente 40 à $50 \%$ de tous les titres publiés annuellement. Dans cette catégorie, le roman occupe une place dominante. Notons que si le nombre d'ouvrages publiés est resté assez stable de 2003 à 2013, la part des ouvrages de langues et littérature est passée de $38 \%$ à $55 \%$. Le genre romanesque semble avoir largement contribué à cet accroissement, puisque la part des romans dans l'ensemble des ouvrages publiés sur la décennie a plus que doublé, passant de 7,7\% en 2003 à plus de $15 \%$ en 2013. En 2013, le roman représentait plus du quart des ouvrages (27\%) de la catégorie langues et littérature, contre un cinquième en 2003.

C'est finalement en consultant la base de données Électre/Memento que nous avons sélectionné les ouvrages de notre corpus. Nous avons d'abord obtenu une liste de 70 ouvrages anglo-canadiens publiés entre 2008-2012. Toutefois, afin d'obtenir l'échantillon le plus diversifié possible, nous avons dû étendre la période de 2003 à 2012. Les quatre romans retenus sont:

- The Speaking Cure de David Homel (Vancouver, Douglas \& McIntyre, 2003) traduit par Lori Saint-Martin et Paul Gagné et publié au Québec la même année sous le titre L’Analyste par Leméac, en coédition et coimpression avec Actes Sud;

- De Niro's Game de Rawi Hage (Toronto, Anansi Press, 2006) traduit par Sophie Voillot pour les éditions Alto au Québec sous le titre de Parfum de poussière en 2007. Cette traduction a été coéditée par Denoël en 2008 sous le titre original, De Niro's Game;

- PlayerOne, What is to Become of us de Douglas Coupland (Anansi Press, 2010) traduit par Rachel Martínez et publié en coédition par Hurtubise HMH au Québec et Au diable vauvert en France sous le titre de Joueur_1 en 2012;

- The Book of Negroes de Lawrence Hill (HarperCollins, 2011) traduit par Carole Noël, publié au Québec par Les Éditions de la Pleine Lune en 2011, puis en France par Présence Africaine Éditions en 2012, sous le titre d'Aminata.

Nous avons présenté plus haut les Éditions Leméac et Actes Sud. The Speaking Cure/L'Analyste raconte l'histoire d'un psychologue dont les compétences sont réquisitionnées par un nouveau régime yougoslave pour traiter les chocs post-traumatiques de rescapés de la ligne de front. La guerre, la dictature, la politique et les amours interdites figurent parmi les concepts culturels mobilisés. L’Analyste est le seul de nos quatre cas dans lequel les deux éditeurs partagent non seulement la traduction, mais l'ensemble du travail de composition et d'impression. Autrement dit, sur les deux territoires, c'est rigoureusement le même livre qui est diffusé, sous des numéros ISBN distincts. 
La deuxième œuvre sélectionnée, De Niro’s Game/Parfum de poussière, a été publiée par la maison Alto de Québec et coéditée en France par Denoël. Alto est une maison littéraire fondée à Québec en 2005 par Antoine Tanguay. Il s'agit d'un éditeur indépendant qui publie une douzaine de titres par année, essentiellement des romans et des nouvelles d'auteurs québécois et des traductions d'auteurs anglophones du Canada et d'ailleurs. Denoël a été fondée à Paris en 1930 et publie de la littérature du monde entier. Cette maison affiliée au groupe Gallimard lance autour d'une centaine de titres par année. D’après le catalogue de Denoël consulté en ligne, De Niro’s Game/ Parfum de poussière est la première expérience de coédition entre les deux maisons qui ont ensuite coédité deux autres œuvres de Rawi Hage. Cette œuvre de fiction porte sur la guerre et le désœuvrement, la violence et la pauvreté matérielle qu'elle engendre dans le contexte géographique du Moyen-Orient. La culture religieuse et les coutumes de la région engendrent également une forme de misère affective et sexuelle dont souffrent les deux principaux personnages du roman. Ce titre a reçu un bon accueil critique et a valu à son auteur le prix IMPAC Dublin (dans sa version originale) et le Prix des libraires du Québec (en traduction française).

La maison d'édition du troisième roman à l'étude, Hurtubise $\mathrm{HMH}$, a été fondée à Montréal en 1960. Son catalogue de plus de 1000 titres comprend des œuvres de fiction mais également de nombreux livres pratiques et de fréquentes coéditions. PlayerOne/Joueur_1 a été coédité avec la maison française Au diable vauvert, fondée en 2000 à Vauvert dans le sud de la France. Le roman de Coupland porte sur la dépersonnalisation à l'ère des médias numériques et de l'individualisme ambiant. Sa publication chez Anansi Press s'inscrit dans le cadre de l'association de cette maison avec les Conférences Massey de l'Université de Toronto, conférences qui ont pour mission de donner une tribune aux penseurs contemporains abordant les problèmes de notre temps. Son auteur, Douglas Coupland, également plasticien et designer, est à l'origine de l'expression "Génération X».

Le dernier ouvrage à l'étude a été publié par Les éditions de la Pleine lune, une maison créée en 1975 et initialement consacrée aux publications féministes. Depuis sa fondation, la Pleine lune a diversifié son catalogue et publie aujourd'hui surtout des romans. Présence Africaine Éditions a été créée en 1949. Cette maison parisienne publie essentiellement des auteurs noirs. Aminata est la seule œuvre à avoir été coéditée entre les deux maisons d'édition. The Book of Negroes/Aminata retrace la vie d'Aminata, une esclave africaine enlevée au Mali qui deviendra, entre les plantations de Caroline du Sud et la Nouvelle-Écosse, une femme admirable en dépit d'un destin tragique. Best-seller au Canada et au Québec, The Book of Negroes/Aminata a obtenu un succès mitigé en France où seuls quelque 1000 exemplaires se sont écoulés. Nous ne pouvons expliquer la panne de succès du livre dans la francophonie hors Québec, mis à part le fait que le caractère spécialisé de la maison d'édition a peut-être contribué à limiter les possibilités commerciales de ce roman grand public.

\subsection{La démarche de recherche}

L'enquête repose sur une méthodologie mixte alliant l'analyse des textes retenus et des entretiens avec ceux et celles qui les ont traduits et publiés au Québec. Si la première démarche (analyse des traductions) visait à mesurer la nature et l'ampleur des modifications apportées aux traductions en vue de leur publication pour le marché 
français, les entretiens ont permis, d'une part, d'obtenir des données contextuelles sur le projet de coédition (données qu'il aurait été impossible de dégager autrement) et, d'autre part, de mieux cerner le point de vue des professionnels engagés dans ces projets. L'analyse textuelle a consisté à repérer tout d'abord, dans les textes originaux, les éléments à forte connotation culturelle et les particularités de ces textes sur le plan littéraire afin de saisir les éventuelles difficultés liées à leur transfert linguistique et culturel. Nous avons ensuite lu les premières éditions françaises afin de dégager les principales stratégies utilisées par les traducteurs (en relation notamment avec le transfert des éléments culturels et la réactivation des spécificités formelles et littéraires du texte). Enfin, nous avons comparé chaque édition québécoise avec l'édition coéditée en France afin de recenser les révisions apportées et autres déplacements que nous avons classés en différentes catégories basées sur la liste des «tendances déformantes» élaborée par Antoine Berman (1986): ennoblissement, appauvrissement, faux sens, pertes, calques et anglicismes, déplacements culturels, pertes d'idiomaticité, etc.

Nous avons mené des entretiens avec les cinq traducteurs des romans retenus ainsi qu'avec trois des quatre éditeurs de tête. Ces entretiens se sont déroulés sous forme de questions ouvertes. Ils ont été enregistrés, puis transcrits et la transcription a fait l'objet d'une codification. En partant du cas à l'étude (soit le titre retenu), nous avons par la suite posé des questions plus générales sur l'expérience que les professionnels interviewés avaient de la coédition. Par exemple, aux éditeurs, nous avons demandé comment ils définissaient la coédition, les raisons pour lesquelles ils y avaient recours, le genre littéraire auquel cette pratique s’appliquait le plus; si le coéditeur demandait parfois des changements aux traductions, et dans l'affirmative, de quelle nature étaient généralement ces changements. Nous avons demandé aux traducteurs si le fait de savoir que leur traduction serait publiée en France avait un impact sur leurs choix de traduction, si les éditeurs leur donnaient parfois des conseils et comment ils définissaient la notion de «français international». Le travail de codification a permis de faire émerger des liens entre les concepts abordés par les personnes interviewées, des constantes, mais aussi des différences dans les discours.

\section{Les traductions et leurs auteurs - À propos du Mid-Atlantic French}

\subsection{Quelles normes de traduction?}

Avant d'analyser les révisions apportées au cours du processus de coédition, il convient de mentionner que ces quatre traductions en elles-mêmes sont assez différentes les unes des autres dans leur rapport à la norme linguistique et au Mid-Atlantic French. Par exemple, dans l'Analyste on trouve peu, voire pas du tout de québécismes. Les traducteurs connaissaient d'emblée l'existence de la coédition et ils ont de toute évidence écrit pour les deux marchés, québécois et français. Leur norme linguistique est internationale. Le roman se déroule à Belgrade, ce qui exclut des réalités culturelles proprement québécoises qui auraient pu entraîner des québécismes. La situation des protagonistes du roman de David Homel tourne autour de la psychiatrie, de la médecine légale et d'une maladie rénale bien spécifique. Outre le vocabulaire médical, le vocabulaire politique, surtout en ce qui touche la dictature, la répression, la guerre et le socialisme, est omniprésent. Les traducteurs sont restés près du texte source et leur projet de traduction consistait à être compris dans toute la francophonie. Parfum 
de poussière regroupe des concepts culturels similaires à ceux de l'Analyste. Le projet de la traductrice, outre les recherches lexicales et terminologiques requises, se voulait stylistique. Elle a collé au style syncopé de l'auteur, produisant une traduction extrêmement littérale. En entretien, la traductrice a précisé que ce littéralisme était volontaire et visait à rester au plus près possible du souffle de l'original.

Pour sa part, la traductrice de Joueur_1 a usé librement de termes québécois, sans trop se préoccuper visiblement du fait que sa traduction pourrait faire l'objet d'une coédition. D'autre part, le sujet du roman, tournant autour de l'utilisation des nouvelles technologies, se prête à l'emploi de néologismes québécois (qui ont, en quelque sorte, fait la norme en ce domaine grâce au travail de l'Office québécois de la langue française) comme équivalents à de nouvelles réalités américaines. De plus, la traductrice a dû faire face à la création pure de concepts par l'auteur Douglas Coupland dont elle a choisi de traduire intégralement les termes. Pour Aminata, la traductrice Carole Noël a adopté les normes stylistiques et lexicales dites «internationales», évitant tout régionalisme, même si l'action du roman se déroule en plus grande partie en Amérique du Nord. De plus, devant les dimensions historiques du sujet et la psychologie des personnages, la traductrice a étudié les mêmes ouvrages que l'auteur Lawrence Hill avait utilisés dans sa recherche, mais dans leurs versions françaises quand elles étaient disponibles, afin, justement, de rendre un français le plus international possible. Dans Aminata, le contexte de l'esclavage a également constitué une difficulté de traduction de nature terminologique. Les niveaux de langue de la narratrice ont également constitué un défi. Il en résulte pourtant une traduction en français assez normatif, dont le lexique élaboré laisse peu de place aux variétés régionales ou sociolectales.

\subsection{Comparaison des éditions québécoises et françaises}

La comparaison des éditions québécoise et française des quatre traductions à l'étude corrobore notre intuition quant à la grande disparité des pratiques de coédition, d'un titre à l'autre. L'Analyste étant un cas de coimpression, le livre est rigoureusement le même pour les deux marchés. Autrement dit, aucun changement n'a été apporté. Pour ce qui est d'Aminata, les demandes du coéditeur, Présence Africaine, sont pour le moins minimales et se résument surtout à des points de ponctuation et de majuscules/ minuscules en plus de la correction de quelques coquilles. Présence Africaine a par exemple demandé la suppression de guillemets fermants au milieu d'un dialogue et l'ajout de la majuscule, surtout des points cardinaux ${ }^{10}$. Les changements de cet ordre, demandés par l'éditeur français, ont été acceptés par l'éditeur de tête. Ils sont au nombre de 33. Deux changements d'ordre rédactionnel, portant sur les extraits suivants, ont été refusés par la Pleine lune. Dans les deux cas, il s'agissait de modifier des temps de verbe.

\section{Changement $\mathbf{n}^{\circ} \mathbf{1}$}

Éditions de la Pleine lune: Je bouillais d'impatience en entendant Clarkson répéter que «des circonstances terribles, tragiques ont entraîné la mort de deux Néo-Écossais respectés, [...].» (p. 471)

Présence Africaine: Je bouillais d'impatience en entendant Clarkson répéter que «des circonstances terribles, tragiques avaient entraîné la mort de deux Néo-Écossais respectés, [...].» (p. 471) 
Justification du refus par l'éditrice de la Pleine Lune: «Il faut absolument garder: ont entraîné, car ce sont les paroles de Clarkson qui sont rapportées telles quelles. Quand il a pris la parole, il a certainement dit: ont été, pour faire référence à l'événement qui avait eu lieu.» (Raoult: 2013)

\section{Changement $\mathbf{n}^{\circ} 2$}

Éditions de la Pleine lune: Ici c'est bien différent: (...). (p. 539)

Présence Africaine: Ici, c'était bien différent: (...). (p. 539)

Justification de l'éditrice: «Il faut absolument garder le présent Ici, c'est bien différent, car on est dans le présent d'Aminata, le moment où elle écrit à Londres. Ce présent est important, car elle parle du village où elle a été recueillie (passé plus lointain) et des jours précédents à Londres (passé plus récent), mais elle est toujours à Londres, au moment présent où elle écrit.» (Raoult 2013)

Pour ce qui est de Joueur_1, le coéditeur français a procédé à quelques changements d'ordre essentiellement lexical. On a remplacé, par exemple, les bas-culottes par des collants, la vadrouille par la serpillière, l'auto par la voiture, les collations de bar par des biscuits apéritifs, le gruau par le porridge, etc. Il s'agit là de changements que l'on pourrait qualifier de culturels, portant sur des notions et réalités de la vie quotidienne qui s'expriment différemment de part et d'autre de l'Atlantique. Par exemple, cahier publicitaire dans la version québécoise devient prospectus en France; quatrième secondaire devient seconde; l'heure du lunch devient l'heure du déjeuner. Ces changements sont tout au plus au nombre d'une dizaine. Par ailleurs, les nombreux termes plus spécialisés présents dans l'original, termes relatifs aux nouvelles technologies, ne sont pas rendus de la même façon dans les deux éditions. Alors que la traductrice québécoise a privilégié des équivalents en français, le coéditeur a plutôt choisi de retourner à la dénomination d'origine, en langue anglaise. Par exemple, la traductrice québécoise utilise le terme courriels et l'éditeur français mails; au Québec, on utilise un cellulaire et en France, un portable. D'autres changements sont plus généraux et, pour la plupart, courants: le stationnement devient un parking en France; une métamorphose beauté, un relooking; le nettoyeur, le pressing. Ce trait renvoie sans doute, là encore, à une différence plus générale d'ordre culturel quant au traitement des nouveaux termes, le Québec pratiquant, depuis plusieurs décennies, une politique systématique de francisation terminologique qui n’a pas d'équivalent en France. Bien sûr, cette politique influence dans une large mesure la pratique des traducteurs. Un autre type de changement est le remplacement de commérer [gossiping] par médire dans l'édition française, changement qui peut être interprété comme une forme d'ennoblissement ou de glissement de sens. Autre exemple possible d'ennoblissement: le terme amygdale au Québec, remplacé par corps amygdaloïde dans la version française. Enfin, on relève une dernière catégorie de changements d'ordre non plus lexical, mais aussi syntaxique et stylistique. Ce groupe inclut, par exemple, le remplacement du syntagme verbal ça goûte + complément - où le sujet est passif et correspond à la chose goûtée plutôt qu'au goûteur - par la formule avoir le goût de dans la version française. La première tournure n'est pas répertoriée dans le TILF et apparaît comme un québécisme dans le Multidictionnaire. On peut donc interpréter son élision comme relevant d'une forme de standardisation guidée par une recherche d'idiomaticité (auprès du lectorat français). L'ensemble des changements relevés, 87 dans un roman de 254 pages, ne touche somme toute qu'une surface limitée du texte. 
Finalement, la traduction du roman de Rawi Hage, Parfum de poussière, a subi davantage de transformations dans l'édition française que les romans précédents. Le style de Rawi Hage est très direct, économique, sans fioriture. Alors que la traductrice québécoise était restée au plus près du texte, optant pour une approche littéraliste, les interventions de l'éditrice de Denoël ont, dans l'ensemble, pour effet d'enrober et d'ennoblir le texte. Outre le remplacement du passé composé de narration par du passé simple - choix lourd de conséquences puisqu'il touchera quasiment chaque page et chaque phrase du livre -, la coéditrice transforme le bruit de la moto en vacarme [...the noise of George's motorbike]. Dans le même esprit, les dents croches [crooked teeth] deviennent des crocs acérés, le passage des voitures [passing cars] devient le trafic. Enfin, la musique arabe [Arabic Songs] laisse place, dans la version coéditée, à des rythmes orientaux aux connotations bien différentes, mais à la fois plus familières et rassurantes, car exotisantes. L'exemple suivant est assez représentatif de ce qui s'observe à l'échelle du roman:

Rawi Hage: [...] the impoverished women carefully, economically, dripped water from red plastic buckets [...]. (p. 14)

Sophie Voillot pour Alto: [...] leurs femmes maigres vidaient avec parcimonie sur leur peau brune des seaux d'eau en plastique rouge [...]. (p. 16)

Sophie Voillot pour Denoël: [...] leurs femmes efflanquées versaient avec parcimonie et prudence un peu d'eau de leur seau en plastique rouge [...]. (p. 16)

S’ils contribuent à ennoblir le texte, certains des changements apportés entraînent aussi des appauvrissements sémantiques ou des glissements, voire des faux sens. C'est le cas, par exemple, lorsque les mots persona [persona] et blanches bacchantes retombantes [droppy white mustache] sont remplacés par des équivalents plus génériques comme personnages et moustache blanche tombante, ou lorsque la race précise d'un chien [poodle] mentionnée dans l'original et dans la traduction, est omise et remplacée par le générique chien chez l'éditeur français. Dans le même ordre d'idées, ôte ta main de sur moi [take your hand off me] devient lâche-moi la main, qui n'a pas tout à fait le même sens; les lésions [bruises] deviennent des plaies et un personnage dans la fin quarantaine, début cinquantaine [early fifties] a plutôt, dans l'édition française du livre, entre quarante et cinquante ans. Ce dernier exemple laisse penser que l'éditrice a parfois privilégié l'idiomaticité et la fluidité - la deuxième expression étant à la fois plus concise et plus idiomatique - au détriment de l'exactitude. Pour l'éditeur d'Alto, ces nombreuses interventions relèvent d'une véritable entreprise de «réécriture» entièrement subjective et qui, selon l'éditeur de tête (Tanguay 2013), n'était nullement nécessaire. Même une rencontre avec ses partenaires à Paris n'a pas permis à cet éditeur de convaincre Denoël de minimiser les changements proposés, changements qui ont heurté la traductrice. "Quand j'ai vu l'ampleur des changements, je n’ai pas été plus loin que le premier chapitre» (Voillot 2013). Malgré tout, par une ironie du sort, la mention «traduit de l'anglais (Canada) par Sophie Voillot» figure sur la page couverture de l'édition de Denoël mais non sur celle des éditions Alto. Dans les liminaires de la coédition française, on mentionne que la traduction est "publiée en accord avec les Éditions Alto», mais aucune mention n'est faite du travail de révision. Les années suivantes, les deux partenaires ont coédité un autre roman de Hage également traduit par Sophie Voillot et, cette fois-ci, l'échange s'est produit sans heurt. Cela semble suggérer que les expériences «difficiles» n’ont pas nécessairement d'impact à moyen terme sur la capacité des éditeurs à travailler ensemble. 
Ces études de cas montrent que les stratégies de traduction observées lors de l'étude réalisée aux éditions du Boréal (Buzelin 2006) ne se vérifient pas dans tous les cas analysés ici. Certains traducteurs, comme Paul Gagné et Lori Saint-Martin, semblent effectuer une forme d'autocensure, privilégiant d'emblée une langue aussi neutre que possible:

Dans le cas de «bleuet» avec Lori, on y va pour petits fruits. Dans un dialogue, mettre dans la bouche d'un Québécois des myrtilles, ça ne se dit pas. Mais il faudrait mettre une note à ce moment-là parce qu'ils vont se demander pourquoi on mange de la confiture de fleurs parce que les bleuets pour (les Français), c’est des fleurs. (Gagné 2013)

D’autres, en revanche, n'hésitent pas à employer des expressions ou des termes clairement identifiables comme étant québécois. Certaines différences à ce titre semblent liées aux concepts culturels ou au cadre spatiotemporel de l'intrigue du roman. L'éditrice Marie-Madeleine Raoult donne un bon exemple où un terme local serait approprié en faisant référence à la traduction en français d'un roman du Montréalais Trevor Ferguson:

(...) mais (dans ce roman) les policiers de Montréal, ils vont prendre des chocolatines à 6 heures du matin avant de prendre, tsé leur...des chocolatines...jamais vu un policier aller prendre des chocolatines! Ils vont peut-être aller prendre un beignet ou un beigne chez Tim Horton, mais ils ne vont pas aller prendre une chocolatine. (Raoult 2013)

Cela dit, tous les traducteurs n'optent pas pour la même norme linguistique et cette diversité se reflète également dans la façon dont ils rendent compte de leur pratique. En effet, les traducteurs dont les textes ont subi le moins de modifications (et occasionnellement leurs éditeurs) sont aussi ceux qui, dans leur discours, minimisent le plus les différences régionales et qui admettent volontiers privilégier une norme internationale. Ainsi, Lori Saint-Martin et Paul Gagné, traducteurs qui ont à leur actif plus de 70 titres, dont plusieurs publiés en coédition, disent tenter d'éviter non seulement les sociolectes existant de part et d'autre de l'Atlantique, autant l'argot français que le joual, mais aussi les régionalismes lexicaux. Ils estiment que les changements demandés par les coéditeurs touchent tout au plus une cinquantaine de termes, toujours les mêmes, et qu'ils n'affectent qu'une petite surface des textes. Ils donnent comme exemple variety store qu'ils rendront par magasin du coin plutôt que par dépanneur. Pour eux, le Mid-Atlantic French n'existe pas vraiment et il n'y a qu'une langue française internationale avec, peut-on conclure implicitement, des différences relativement superficielles et minimes. Pour sa part, l'éditrice d'Aminata, Marie-Madeleine Raoult regrette la tendance consistant à modifier les modes d'expression et à les édulcorer pour plaire à l'autre: «Je pense que c'est maladroit de vouloir changer ce qu'on est pour être accepté, ben je pense que dans la littérature, c'est pareil» (Raoult 2013). Elle ajoute toutefois que «la langue française est partout la même». Contrairement aux traducteurs du roman de David Homel, la traductrice d'Aminata, Carole Noël, ignorait si le roman serait publié en France au moment où elle s'est mise au travail et affirme que le contraire n'aurait pas influencé pour autant ses choix de traduction ni son texte final. En effet, dans un cas comme dans l'autre, la traductrice évite l'emploi de québécismes, sauf si le contexte culturel l'exige. De son propre dire, c'est ainsi qu'elle s'épargne des demandes de changements de la part de l'éditeur français.

La perception de la traductrice de Joueur_1, Rachel Martínez, diffère nettement de celle qui émanait des traducteurs précédents. En effet, alors que les premiers tendaient 
à minimiser l'influence du lectorat (projeté) sur leur pratique, cette traductrice affirme s'interroger sur le récepteur de sa traduction, et ce, avant même de commencer son travail. D’emblée, elle admet que ses choix seront influencés par la possibilité que le roman soit vendu en France. Pourtant, dans le cas étudié ici, elle reconnaît ne pas s'être particulièrement attachée à la question de la diffusion internationale, pour se concentrer plutôt sur le ton à employer dans le cas du roman de Coupland où cinq voix se succédaient. Quant à Sophie Voillot, elle se plonge dans les univers culturels des romans qu'elle a à traduire tout en mettant la voix de l'auteur à l'avant-plan. Pour elle, l'autocensure du traducteur pour atteindre un français dit international dépend de l'œuvre à laquelle il se mesure. Pour elle, le Mid-Atlantic French est une pratique plus qu'un concept.

J'écris en français avec les règles de la langue. Le reste relève davantage de la sociologie de la traduction, en quelque sorte. La culture et le contexte du roman vont orienter le choix des termes. C’est une notion qu’on ne peut pas vraiment définir. (Voillot 2013)

\section{4. "Ils ont toujours raison" - La coédition, la domination culturelle et l'idéologie}

Les expériences de coédition - quelles que soient leurs modalités et les langues concernées - comportent généralement une dose d'imprévisibilité liée aux délais de production et autres contraintes qui peuvent empêcher l'une ou l'autre partie de respecter toutes les modalités du contrat. Les ententes de coédition France/Québec n'échappent pas à la règle. Par exemple, alors que ces ententes prévoient l'accord des éditeurs et traducteurs québécois pour les changements que le coéditeur souhaite apporter aux traductions, dans la pratique, selon les éditeurs interviewés, ces dispositions contractuelles ne sont pas toujours respectées. Arnaud Foulon cite le cas du roman Joueur_1:

Non, nous ce qu'ils ont fait, c'est qu'ils nous ont expliqué la démarche qu'ils allaient appliquer dans le cadre d'un courriel, je me rappelle que l'exemple parking-stationnement c'en est un qui nous a été mentionné, mais ils ne nous ont pas montré chaque changement sur chaque page qu'ils ont fait, alors qu'ils devraient le faire. (Foulon: 2013)

Si ces «irrégularités» ne sont pas forcément intentionnelles, les accords de coédition, tout comme les échanges internationaux de livres en traduction, s'inscrivent dans un espace littéraire mondial différencié et hiérarchisé. Plus exactement, ce champ «s'ordonne selon l'opposition entre les grands espaces littéraires nationaux qui sont aussi les plus anciens, c'est-à-dire les plus dotés, et les espaces littéraires les plus récemment apparus et peu dotés " (Casanova 1999: 120). Or, selon l'analyse de Casanova, l'histoire de ces échanges aurait contribué à faire de Paris la capitale de cette République mondiale des lettres:

Paris n'est pas seulement la capitale de l'univers littéraire, il est aussi, de ce fait, la porte d'entrée du «marché mondial des biens intellectuels», comme le disait Goethe, le lieu consacrant majeur du monde de la littérature. La consécration parisienne est un recours nécessaire pour les auteurs internationaux de tous les espaces littéraires dominés: traductions, lectures critiques, éloges et commentaires sont autant de jugements et de verdicts qui donnent valeur littéraire à un texte jusque-là tenu hors des limites de l'espace ou non perçu. (Casanova 1999: 180) 
Dans ce contexte, et sans grande surprise, le discours le plus récurrent qui ressort des entretiens sur la coédition que nous avons conduits auprès des traducteurs et éditeurs québécois tourne autour de la domination culturelle et linguistique de la France sur le Québec, comme si la coédition venait systématiquement rappeler, voire exacerber, ce sentiment de domination.

L'édition en France a un standing, pour eux c'est important de corriger ce que les éditeurs québécois font. (...) Et encore là, un éditeur comme Diable Vauvert, c'est pas un éditeur comme Grasset ou le Seuil... où là eux ont souvent des impressions de grandes maisons françaises ou eux, ont tendance à encore plus intervenir. Parce que je pense que ça vient de la base qu'ils n’ont pas nécessairement confiance aux professionnels de l'édition au Québec... moi je dis ça, eux répondraient pas ça, mais moi c'est ce que je dis. (Foulon 2013)

Selon certains des éditeurs et des traducteurs qui ont participé à cette étude, les zones de tension se manifestent en particulier lorsque vient le temps de traduire une œuvre de fiction dont l'intrigue se déroule au Canada ou au Québec, ou lorsque les personnages emploient des registres de langue familiers.

Dans une de mes traductions à venir, il y aura le mot «bécosse». J'espère qu'ils ne vont pas me changer ça pour... enfin. Dans cette culture générale, les Français croient que les Québécois sont un peuple de bûcherons et de paysans mal dégrossis. Le Québec... une culture dominée chez elle et en France. (Voillot 2013)

Sophie Voillot croit que la langue française au Québec est doublement marginalisée, à la fois par le reste du Canada et par la France «qui la prend de haut». De son côté, Rachel Martínez donne en anecdote le cas d'un coéditeur qui tenait à rendre residential school (on parle ici des pensionnats autochtones) par le calque école résidentielle plutôt que pensionnat indien, attitude ressentie par la traductrice comme une forme "d'impérialisme culturel». "On est habitués nous à avoir toutes sortes de français, les Français eux ils pensent qu'ils ont la vérité.» (Martínez 2013). Cette traductrice racontera avoir entendu un traducteur français exprimer un désintérêt évident pour la culture québécoise. D’autres traducteurs comme Lori St-Martin et Paul Gagné estiment, au contraire, que le complexe de supériorité du milieu littéraire français à l'égard des traducteurs québécois est en train de s'atténuer et que le marché français dans l'ensemble n'est plus réfractaire aux traductions québécoises, même si certains coéditeurs demeurent parfois frileux. Ainsi, la question du rapport de force entre les champs littéraires français et québécois est systématiquement évoquée par les traducteurs, mais les perceptions que ceux-ci ont de la nature de ce rapport ne sont pas pour autant uniformes.

Cette diversité d'opinions n'apparaît pas chez les éditeurs dont le discours sur la coédition semble beaucoup plus homogène. Tous insistent, de différentes façons, sur la domination du champ littéraire français sur celui du Québec. Les éditeurs interviewés ne manquent pas de rappeler que la littérature française jouit d'une reconnaissance beaucoup plus importante que celle du Québec, que les maisons d'édition parisiennes ont une plus grande ancienneté et, à ce titre, ont plus d'influence que les jeunes maisons du nouveau continent; ces éditeurs perçoivent aussi un manque de confiance des éditeurs français à l'égard des maisons d'édition québécoises. Certaines positions sont si catégoriques qu'elles peuvent presque être qualifiées d'idéologiques, comme l'éditeur Antoine Tanguay affirmant, avec ironie, que les éditeurs français, 
«c'est eux autres qui ont raison». Cette nécessité de "céder» dans les négociations trouve une illustration parfaite dans le cas de Parfum de poussière, où l'éditeur et la traductrice ont fini par laisser le coéditeur publier une traduction largement réécrite. Dans l'ensemble, le discours des éditeurs réitère le caractère asymétrique des échanges éditoriaux France/Québec - réalité rappelée et développée également dans le chapitre premier du mémoire de Baju (2004) - où Paris est encore dépeinte comme la capitale mondiale du livre, même si ce titre lui semble de plus en plus contesté11.

En somme, les éditeurs interviewés tiennent un discours assez uniformisé, moins nuancé que celui des traducteurs, discours dans lequel les interventions des coéditeurs français sont systématiquement expliquées en termes de rapports de force entre la France et le Québec. Pourtant, il semble que pour une mauvaise expérience de coédition, beaucoup d'autres se déroulent dans l'harmonie. Nous avons à ce sujet observé une certaine ambivalence chez les éditeurs. Si, d'une part, ils déplorent une forme de condescendance de la part de leurs vis-à-vis français, ils souhaitent quand même s'associer à eux, surtout s'ils jouissent d'une certaine importance, afin d'accroître leur notoriété dans le champ littéraire et de faire rayonner leurs œuvres. Sur la question de la norme linguistique - question sensible chez les traducteurs -, la plupart des éditeurs québécois interrogés ici se montrent souples et sont portés à céder sur certains points pour parvenir, sans trop de heurts, à faire rayonner les ouvrages canadiens-anglais dans le reste de la francophonie.

\section{Discussion}

Au-delà de la diversité des scénarios d'un titre à l'autre et du fait que les pratiques ne suivent pas toujours, comme le rappellent les professionnels, les règles stipulées dans les contrats, l'étude révèle un décalage assez fort entre les cas que nous avons répertoriés - des histoires le plus souvent, du moins dans trois cas sur quatre, relativement harmonieuses - et le discours très critique, et parfois à portée idéologique, des éditeurs et, dans une moindre mesure, des traducteurs; un discours axé avant tout sur la réaffirmation des rapports de domination culturelle et linguistique de la France vis-à-vis du Québec. Ce décalage peut s'expliquer de plusieurs façons. Certains y verront l'expression d'une insécurité linguistique, du sentiment d'une absence de reconnaissance de la légitimité de la norme québécoise en traduction. Cette notion d'insécurité linguistique a été étudiée par Chantal Bouchard (2002) dans une perspective historique. Elle retrace, entre autres, les différentes études, cadres législatifs et outils dont se sont dotés les Québécois au fil du temps pour définir, circonscrire et protéger leur intégrité linguistique.

L'histoire du français au Québec est l'histoire d'une langue dominée. Le discours métalinguistique pourrait être qualifié de discours méta-identitaire. En parlant de leur langue, les Québécois se sont décrits eux-mêmes. (Bouchard 2002: 287)

Elle conclut que la préoccupation québécoise pour la langue est étroitement liée à une quête d'identité profonde.

L'orientation des actions collectives des Québécois depuis la Révolution tranquille va systématiquement dans le sens d'une revalorisation de leur langue et de leur culture, à leurs yeux d'abord, mais aussi vis-à-vis des autres sociétés qui tiennent un rôle de référence important: les Anglo-Canadiens, la société nord-américaine dans son ensemble, la France et la francophonie en général. (Bouchard 2002: 286) 
Notre recherche a surtout mis en lumière les zones de tension entre les champs littéraires québécois et français, tensions qui s'exercent sur fond de perception de déficit du capital symbolique littéraire des éditeurs québécois. Ainsi, les discours analysés ici rappellent à quel point la vie et les milieux intellectuels n'échappent pas à ces formidables effets de clôture qui caractérisent tous les espaces sociaux:

La vie intellectuelle est le lieu, comme tous les espaces sociaux, de nationalismes, et les intellectuels véhiculent, presque autant que les autres, des préjugés, des stéréotypes, des idées reçues, des représentations très sommaires, très élémentaires, qui se nourrissent des accidents de la vie quotidienne, des incompréhensions, des malentendus, des blessures (celle par exemple que peut infliger au narcissisme le fait d'être méconnu dans un pays étranger). (Bourdieu 2002:3)

Offrant aux traducteurs, auteurs et éditeurs la perspective d'un plus grand rayonnement, la coédition est aussi le lieu où se jouent, de part et d'autre, ces rapports de force, ces préjugés, stéréotypes et nationalismes. Par exemple, les rapports de force entre les éditeurs peuvent s'exercer dans la négociation et le partage des territoires; les préjugés de part et d'autre du processus de coédition teintent souvent un discours largement empreint de perceptions; les stéréotypes à l'égard des attentes du lectorat influencent à divers degrés les choix de traduction faits par les traducteurs; les nationalismes, plus ou moins marqués selon les marchés dans lesquels sont invités à agir les partenaires, viennent parfois nourrir des frictions. Par ailleurs, on remarque que le discours des traducteurs est plus diversifié que celui des éditeurs. En effet, tandis que certains insistent sur la difficulté de percer le marché français, d'autres considèrent que les traductions québécoises y sont plutôt bien reçues. Bien que le nombre de traducteurs consultés soit limité, il semble exister un lien entre les pratiques de ces professionnels, leur discours et la place qu'ils occupent dans le champ de la traduction littéraire au Québec ${ }^{12}$. Autrement dit, le discours que les traducteurs portent eux-mêmes sur leur pratique reflète des modes de différenciation et des hiérarchies bien réelles au sein de l'activité. Tout comme en France, on relève tout d'abord deux profils de traducteurs: ceux qui tirent leur principale source de revenus de la traduction (et qui pratiquent souvent la traduction pragmatique parallèlement à la traduction littéraire) et ceux qui occupent d'autres positions dans les champs littéraire ou universitaire (directeurs de l'édition, professeurs, auteurs, etc.). Si les premiers ne sont pas nécessairement dans une position économique plus précaire (contrairement à ce qu'a pu observer Kalinowski [2002)] pour la France), les seconds sont en revanche susceptibles d'être mieux dotés en capital social et culturel.

Parmi les traductrices rencontrées dans cette étude, deux tiraient essentiellement leurs revenus de la traduction, tandis que les autres avaient aussi des liens avec les sphères littéraire ou universitaire. Par ailleurs, on observe une hiérarchisation qui recoupe largement celle des genres. Au sommet se trouve la traduction des œuvres de fiction des auteurs du canon, à l'opposé, celle des livres jeunesse (sans compter les ouvrages à caractère général ou didactique que les traducteurs préféreront parfois signer sous un autre nom). Ainsi, une traductrice nous a confié qu'au terme d'une période pendant laquelle elle avait traduit des essais et des livres pour enfants, le milieu littéraire avait eu tendance à l'oublier. Le type d'ouvrages traduits contribue donc à structurer le champ et à délimiter les positions qu'y occupent ceux qui le constituent, tout comme la consécration par les prix de traduction, même si l'impact 
de ceux-ci sur la visibilité des traducteurs auprès du grand public reste bien limité (voir Zapata et Le Plouhinec 2015).

Des quatre cas étudiés, deux constituent des coéditions «sans histoire» pour lesquelles il n'y a eu que très peu, voire aucun changement apporté. Les auteurs de ces traductions sont ceux qui avaient la plus longue expérience du métier, ceux qui tendaient à minimiser les difficultés dues aux variations linguistiques régionales, qui admettaient volontiers traduire dans une «langue internationale» et dont les traductions contiennent, de fait, le moins de traces de leur contexte d'énonciation. On peut supposer que ces traducteurs, dont certains occupent une position clairement dominante dans le champ (par leur ancienneté, la nature des ouvrages traduits, le type et le nombre de prix reçus, etc.) ont intégré un ensemble de normes correspondant à la fois aux attentes de leurs donneurs d'ouvrage et à celles d'un éventuel coéditeur français, les deux étant d'ailleurs étroitement liés (voir Buzelin 2005). Ces traducteurs tendent à anticiper les difficultés et ne voient pas dans la coédition un enjeu qui modifiera leur pratique. Sans nier l'existence de rapports de force entre le marché français et le marché québécois, ils tiennent un discours assez positif et mettent l'accent sur les opportunités de la coédition et sur l'ouverture des éditeurs français et de leurs marchés aux traductions québécoises. Dans les deux autres cas, engageant non seulement une relecture, mais des changements tantôt superficiels tantôt majeurs de la part du coéditeur, l'inégalité des rapports de force était dénoncée beaucoup plus vivement par les traducteurs. En entretien, la traductrice ayant vu son texte réécrit par la coéditrice française avait peine à aborder le sujet, son mutisme dénotant un certain malaise. Dans ces deux cas, tout en mentionnant parfois l'importance d'adapter la traduction au lectorat, les traductrices semblaient avoir beaucoup moins «joué le jeu» du Mid-Atlantic French. Cette différence peut être en partie liée au positionnement des traductrices qui, contrairement à celles des cas précédents, traduisent aussi des textes pragmatiques et ont un profil plus professionnel qu'universitaire.

\section{Conclusion}

Le développement des ententes de coédition France-Québec vers la fin du siècle dernier s'est accompagné d'un discours public très positif de la part des professionnels québécois qui voyaient dans ces partenariats une bonne façon de percer le marché français et de faire rayonner la littérature domestique, qu'il s'agisse d'auteurs québécois ou canadiens (Buzelin 2009), autrement dit « une solution d'avenir» (Baju 2004). Ce discours diffère nettement de celui, beaucoup plus critique, voire parfois idéologique qui, une vingtaine d'années plus tard, émane de nos entretiens avec les éditeurs, un discours axé sur la réitération de la domination linguistique et culturelle de la France sur le Québec. Ce décalage peut être perçu en partie comme l'expression d'une certaine déception, comme si les attentes n’avaient pas été remplies. Car comme le notait Baju:

Les éditeurs québécois ne s'en cachent pas: leur objectif premier, lorsqu'ils se lancent dans la diffusion de leurs livres à l'étranger, est le marché français. C'est une ambition propre au champ éditorial québécois pour qui la France représente le plus gros marché du livre francophone; mais également à son champ littéraire qui regarde sans cesse vers le champ littéraire français pour en obtenir la reconnaissance et la consécration. Les éditeurs veulent vendre leurs livres aux Français et les auteurs veulent être lus par les Français. (Baju 2004: 84) 
La difficulté à atteindre cet «objectif premier» peut expliquer aussi en partie le décalage entre le discours des éditeurs québécois, un discours mettant l'accent sur la domination du champ littéraire québécois, et les expériences relatives aux quatre cas étudiés, lesquels lèvent le voile sur une réalité moins sombre qu'il n'y paraît puisque, la plupart du temps - dans trois titres sur quatre -, ces coéditions se sont bien passées et ont occasionné des modifications somme toute assez mineures. La coédition semble donc plus complexe et problématique dans le discours que dans les pratiques. Elle devient un objet privilégié du discours des éditeurs qui y voient un sujet complexe: un reflet des rapports de domination en même temps qu'une opportunité d'affirmer le professionnalisme et la légitimité de l'édition et de la traduction au Québec.

Dans les faits, si l'on se fie aux cas étudiés - engageant des auteurs, des maisons, des coéditeurs et des traducteurs distincts -, la coédition ne semble pas modifier en profondeur les pratiques de traduction, car elle touche le plus souvent des aspects limités du texte, tant sur le plan qualitatif comme le lexique (plutôt que la syntaxe) que sur le plan quantitatif puisque les termes problématiques ne sont pas si nombreux que cela. Ainsi, les données ne nous permettent pas de conclure à l'existence d'une langue de traduction "aseptisée» qui gommerait toute trace du lieu de son énonciation. Cela revient à confirmer aussi que le Mid-Atlantic French renvoie plus à une posture de traduction qu'à une norme en tant que telle. En revanche, l'analyse de ces quatre cas montre bien des variations importantes dans le rapport des traducteurs littéraires à leur langue et dans les stratégies de traduction utilisées, variations qui semblent en partie liées à la position que ces traducteurs occupent dans leur champ. Ainsi, ceux qui sont en position dominante semblent aussi avoir le mieux intériorisé les attentes de leurs donneurs d'ouvrage (et de leurs éventuels partenaires), au point de pouvoir afficher, dans leur discours, un certain détachement par rapport à ces questions de normes et de lectorats. On pourrait d'ailleurs voir, dans l'intériorisation de ces attentes, un des facteurs de «succès» de ces traducteurs. Se conformant à la norme, ceux-ci contribuent à la renforcer et en retirent les plus grands bénéfices.

Ainsi, le paysage de la coédition est trop hétérogène pour en brosser un portrait monochrome. Le processus est en outre influencé par les relations interpersonnelles des parties en jeu, les contextes mobilisés, le pouvoir réel ou avéré des partenaires et aussi, une bonne dose de subjectivité quant à ce que les lecteurs sont prêts à accepter dans les traductions que leur proposent les éditeurs. Enfin, l'étude montre tout de même que les cas problématiques - qui sont minoritaires - peuvent être extrêmement mal vécus à court terme. Comme le rappellent Lori Saint-Martin et Paul Gagné, «si l'éditeur français a du pouvoir sur l'éditeur québécois, n’importe quel éditeur a plus de pouvoir que n'importe quel traducteur. Il y a plusieurs échelons à l'intérieur de tout cela et le plus bas, c'est le traducteur» (Saint-Martin et Gagné 2013). Le fait que les traducteurs québécois et le coéditeur/réviseur étranger n’aient aucun contact direct - qu'ils ne se connaissent parfois même pas et que 5000 kilomètres les séparent - peut contribuer à exacerber les malentendus, les incompréhensions, les écarts et finalement les rapports de force. Cela dit, les histoires répertoriées ici montrent aussi que l'expérience la plus douloureuse à court terme n'a pas nécessairement d'incidence sur la capacité des partenaires à nouer de nouvelles ententes par la suite.

Cette étude portait sur des traductions réalisées au Québec puis diffusées, sous la marque d'un éditeur français, dans l'Europe francophone. En outre, elle abordait l'objet du point de vue des acteurs québécois seulement. Par souci de symétrie et pour 
obtenir un portrait plus riche et nuancé, il serait intéressant d'étudier à l'avenir à la fois la perspective des acteurs - éditeurs et traducteurs - français du milieu du livre et la façon dont les traductions, effectuées en France et coéditées au Québec, sont éventuellement relues et révisées en vue de leur diffusion dans l'Amérique du Nord francophone. Enfin, on pourrait interroger à quel point les enjeux et les pratiques analysées ici caractérisent aussi les ententes de coédition engageant d'autres langues internationales telles que l'anglais ou l'espagnol. Dans cette optique, il faudrait toutefois garder à l'esprit que les rapports de force entre les langues (ainsi qu'entre les diverses variétés d'une même langue) peuvent être très différents d'un contexte à l'autre.

\section{NOTES}

* Cet article découle d'une recherche de maîtrise en traduction réalisée en 2013-2014. Il reprend une partie des données et résultats que nous avons présentés dans notre mémoire intitulé La coédition franco-québécoise et ses conséquences sur les œuvres de fiction publiées en traduction, disponible à la bibliothèque de l'Université de Montréal (voir Beaulieu 2014). Les auteures remercient les deux évaluateurs de Meta dont les questions et remarques constructives ont permis d'enrichir le texte de plusieurs clarifications, précisions et nuances.

1. En 1985, le Conseil des arts du Canada a lancé un deuxième programme visant cette fois à encourager la traduction de la littérature nationale, par des éditeurs étrangers, dans des langues autres que le français et l'anglais.

2. Par exemple, 81 ouvrages ont reçu des subventions à la traduction en 1998-1999 et 86 en 2007-2008. On note depuis 2009-2010 (117 subventions accordées) une augmentation du nombre de subventions qui atteint 150 en 2013-2014 selon les statistiques du Conseil des arts du Canada.

3. Il s'agit bien sûr d'une tendance qui ne couvre pas tous les cas de figure: il arrive que des auteurs anglo-canadiens soient traduits en France et coédités avec le Québec (ce fut le cas, par exemple, de Michael Ondatjee) et que des ouvrages non admissibles au programme du CAC soient traduits au Québec puis coédités en France.

4. Il s'agissait d'un nouveau roman signé par un auteur anglo-canadien, roman donc admissible au progamme d'aide à la traduction du Conseil des arts et qui, au moment de la cession des droits de traduction, connaissait un bon succès commercial au Canada, aux États-Unis et en Grande-Bretagne.

5. Ainsi, dans l'industrie des manuels destinés à l'enseignement supérieur, l'un des secteurs éditoriaux où le processus de concentration des maisons d'édition touche son paroxysme, ces ententes sont beaucoup plus rares.

6. À ce titre, le partenariat de plus long terme tissé entre les éditions Leméac et Actes Sud, partenariat décrit dans le mémoire de maîtrise de Baju (2004), semblerait constituer une exception, à tout le moins au Québec.

7. Dans nos entretiens avec les éditeurs, nous avons constaté que toute généralisation quant à un modèle de coédition est impossible car il semble exister autant de modèles qu'il y a de coéditions. Certains modèles pratiqués par les éditeurs servent néanmoins de base. Ils regroupent au moins deux éditeurs dont l'entente peut aller de la coproduction avec partage des risques, des coûts et des droits jusqu'à la «simple» cession des droits de traduction pour un territoire particulier, incluant la vente du texte traduit avec ou sans modification (Buzelin: 2006, 161).

8. Nous entendons par là des éditeurs et traducteurs qui élaborent des projets de traduction littéraire entendue au sens donné par le Conseil des arts du Canada (fiction, essai, poésie, théâtre, etc.).

9. Éditions de la Pleine Lune: «Nsé i ni sógóma», répondit-elle en Bambara (p. 150); Présence Aafricaine: «Nsé i ni sógóma», répondit-elle en Bambara (p. 150). 
10. Éditions de la Pleine lune: «Il dit qu'ils se cachaient parmi les Indiens ou partaient vivre avec les Espagnols dans le sud» (p. 190); Présence Africaine: «Il dit qu'ils se cachaient parmi les Indiens ou partaient vivre avec les Espagnols dans le Sud» (p. 190).

11. Notons que si Casanova montre bien comment Paris a pu devenir la «capitale mondiale du livre», elle signale aussi l'existence de signes d'érosion depuis les années 1960 au moins. La question des tensions au sein de la sphère de production restreinte du champ littéraire français est également analysée par Buzelin (2015).

12. La traduction littéraire au Québec semble bien en passe de constituer un champ, au sens sociologique. En effet, si les traductions de livres faites au Québec ne comptent que pour environ $10 \%$ de l'ensemble des ouvrages traduits en français et s'il n'existe pas d'organe officiel représentant les traducteurs littéraires de la province (ceux-ci étant généralement membres de l'Association des traducteurs et traductrices littéraires du Canada), de nombreux indices laissent supposer l'émergence, récente, d'un champ de la traduction à proprement parler: l'augmentation des traductions publiées au cours des trente dernières années, la professionnalisation de la pratique (grâce au soutien du Conseil des arts du Canada), l'attention accrue portée par les critiques et médias à la traduction littéraire, le développement de programmes universitaires (par exemple à l'Université de Sherbrooke), l'existence d'instances de consécration (prix de traduction du Gouverneur général), l'apparition de projets de traduction d'ouvrages étrangers (projets allant outre le cadre dicté par le Conseil des arts). Un autre signe tangible de ce développement est le «rapatriement» du canon littéraire anglo-montréalais, et plus généralement anglo-canadien, grâce à de récentes retraductions, made in Québec, des œuvres de Leonard Cohen, Margaret Atwood et Mordecai Richler, retraductions qui sont toujours annoncées comme des évènements littéraires.

\section{RÉFÉRENCES}

BACON, Josephine (2005) : How co-editions can go wrong: pitfalls of cross-cultural translation. LOGOS. 16(1):48-51.

BAju, Sarah (2004): La coédition: une solution d'avenir? Les relations éditoriales entre la France et le Québec: le cas des éditions Actes Sud et Leméac Éditeur (1989-2003). Mémoire de maîtrise. Sherbrooke: Université de Sherbrooke/Paris: Panthéon Sorbonne.

BeAulieu, Solange (2014): La coédition franco-québécoise et ses conséquences sur les auvres de fiction publiées en traduction. Mémoire de maîtrise. Montréal: Université de Montréal.

Berman, Antoine (1986): La traduction comme épreuve de l'étranger. Texte. 4:67-81.

Bouchard, Chantal (2002): La langue et le nombril, une histoire sociolinguistique du Québec. Montréal: Fides.

Bourdieu, Pierre (1991): Le champ littéraire. Actes de la recherche en sciences sociales. 89:3-46.

Bourdieu, Pierre (2002): Les conditions sociales de la circulation internationale des idées. Actes de la recherche en sciences sociales. 145:3-8.

Buzelin, Hélène (2006): Independent Publisher in the Networks of Translation. TTR. 19(1):135-173.

Buzelin, Hélène (2007): Translations in the Making. In: Michaela Wolf et Alexandra FukARI, dir. Constructing a Sociology of Translation. New York/Amsterdam: John Benjamins, 135169.

Buzelin, Hélène (2007b): Repenser la traduction à travers le spectre de la coédition. Meta. 52(4):688-723.

Buzelin, Hélène (2009): Les contradictions de la coédition internationale: des pratiques aux représentations. In: Gisèle SAPIro, dir. Les contradictions de la globalisation éditoriale. Paris: Nouveau Monde Éditions, 45-79.

Buzelin, Hélène (2015): Traduire pour le Centre national du livre. COnTEXTES. Consulté le 21 décembre 2015, http://contextes.revues.org/6095

Casanova, Pascale (1999): La République mondiale des lettres. Paris: Le Seuil.

Coupland, Douglas (2012) : Joueur_1: ce qu'il adviendra de nous. (traduit de l'anglais par Rachel Martinèz). Montréal: Éditions Hurtubise. 
Coupland, Douglas (2012): Joueur_1. (traduit de l'anglais par Rachel MartinÈz). Vauvert: Éditions Au diable vauvert.

Dollerup, Cay et Orel-Kos, Silvana (2001): Co-prints and Translation. Perspectives: Studies in Translatology. 9:87-108.

FILION, Pierre (1991) : Écrire au Québec, être lu ailleurs. In: Lise Gauvin et Jean-Marie KLINKenBerg, dir. Écrivain cherche lecteur. Paris: Éditions Créaphis/Montréal:VLB Éditeur, 221-227.

ForgEt, Louise (2004) : La coédition... dans une économie de l'instant. Livre d'ici. 204:5-6.

Fouché, Pascal, Péchoin, Daniel, Schuwer, Philippe, dir. (2002) : Dictionnaire encyclopédique du livre. Paris, Éditions du Cercle de la Librairie.

Guardian, The (2006): Dictionary of Publishing and Printing. London: A \& C Black.

Hage, Rawi (2007): Parfum de poussière. (traduit de l'anglais par Sophie Volllot). Québec: Alto.

Hage, Rawi (2008): De Niro's Game. (traduit de l'anglais par Sophie Volllot). Paris: Denoël.

Heinich, Nathalie (1984): Les traducteurs littéraires: l'art et la profession. Revue française de sociologie. 25:264-280.

HıLl, Lawrence (2012): Aminata. (traduit de l'anglais par Carole NoËL). Paris: Présence Africaine éditions.

HıLl, Lawrence (2011): Aminata. (traduit de l’anglais par Carole NoËL). Lachine: Éditions de la Pleine lune.

Homel, David (2003): L'analyste. (traduit de l’anglais par Lori Saint-Martin et Paul Gagné). Montréal: Leméac/Actes Sud.

Kalinowski, Isabelle (2002): La vocation au travail de traduction. Actes de la recherche en sciences sociales. 144:47-54.

Katan, David (2009): Translation Theory and Professional Practice: A Global Survey of the Great Divide. Hermes - Journal of Language and Communication Studies. 11-2:111-53.

RoBin, Christian (2006): Coédition/coproduction, effets sur les contenus et les publications: le cas du livre pratique illustré. Les contradictions de la globalisation éditoriale, École des hautes études en sciences sociales, Paris, 23-25 mars 2006.

SAPIro, Gisèle, dir. (2009): Les contradictions de la globalisation éditoriale. Paris: Nouveau Monde éditions.

SChUWer, Philippe (1991): L'édition internationale, coéditions et coproductions. Paris: Éditions du Cercle de la Librairie.

SERry, Hervé (2006): Faire l'Europe: Enjeux intellectuels et enjeux internationaux d'une collection transnationale. In: Gisèle SAPIRO, dir. Les contradictions de la globalisation éditoriale. Paris: Nouveau Monde éditions, 227-49.

Toury, Gideon (1995): Descriptive Translation Studies and Beyond. Amsterdam/Philadelphie: John Benjamins.

Vaugeors, Denis (2002) : La coédition entre la France et le Québec. Études canadiennes/Canadian Studies. $52: 247-251$.

Zapata, Juan et Le Plouhinec, Valérie (2015): Les prix de traduction fabriquent-ils une image régulatrice du traducteur? Convergences francophones. 2:1-17.

\section{ENTREVUES}

Entrevue avec Arnaud Foulon, éditeur, Montréal, 29 octobre 2013. Enregistrement audio de $34 \mathrm{~min} 59 \mathrm{sec}$.

Entrevue avec Paul Gagné et Lori Saint-Martin, traducteurs, Montréal, 6 septembre 2013. Enregistrement audio de 49 min $21 \mathrm{sec}$.

Entrevue avec Rachel Martinez, traductrice, Montréal, le 25 juillet 2013. Enregistrement audio de 1 h 25 min $59 \mathrm{sec}$.

Entrevue avec Carole NoËL, traductrice, Québec, le 7 juillet 2013. Enregistrement audio de $60 \mathrm{~min}$. 
Entrevue avec Marie-Madeleine Raoult, directrice, Éditions de la Pleine lune, Lachine, 22 juin 2013. Enregistrement audio d'une durée de 1 h 06 min $51 \mathrm{sec}$.

Entrevue avec Antoine TAnguay, fondateur et éditeur, Éditions Alto, Québec, 18 juin 2013. Enregistrement audio d'une durée de $1 \mathrm{~h} 10 \mathrm{~min} 37 \mathrm{sec}$.

Entrevue avec Sophie Volllot, traductrice, Montréal, 13 juillet 2013. Transcription d'un entretien d'environ $45 \mathrm{~min}$.

\section{ANNEXES}

TABLEAU 1

Nombre d'ouvrages déposés à la Bibliothèque nationale du Québec

\begin{tabular}{llllllllllll}
\hline Année & 2003 & 2004 & 2005 & 2006 & 2007 & 2008 & 2009 & 2010 & 2011 & 2012 & 2013 \\
\hline Ouvrages & 6083 & 6387 & 6447 & 6559 & 6274 & 7342 & 7183 & 7157 & 6825 & 6839 & 6323 \\
\hline $\begin{array}{l}\text { Langue et } \\
\text { littérature }\end{array}$ & 2338 & 2678 & 3034 & 3118 & 3060 & 3638 & 3572 & 3621 & 3617 & 3861 & 3484 \\
\hline Romans & 472 & 557 & 622 & 602 & 575 & 800 & 808 & 819 & 802 & 875 & 972 \\
\hline
\end{tabular}

Source: $B A N Q$.

TABLEAU 2

Traductions publiées au Canada

\begin{tabular}{llllllllllll}
\hline Année & $\mathbf{2 0 0 9}$ & $\mathbf{2 0 0 8}$ & $\mathbf{2 0 0 7}$ & $\mathbf{2 0 0 6}$ & $\mathbf{2 0 0 5}$ & $\mathbf{2 0 0 4}$ & $\mathbf{2 0 0 3}$ & $\mathbf{2 0 0 2}$ & $\mathbf{2 0 0 1}$ & $\mathbf{2 0 0 0}$ & $\mathbf{1 9 9 9}$ \\
\hline Ouvrages & 323 & 1606 & 1971 & 2083 & 1684 & 1362 & 1515 & 1166 & 1172 & 1298 & 1528 \\
\hline Année & $\mathbf{1 9 9 8}$ & $\mathbf{1 9 9 7}$ & $\mathbf{1 9 9 6}$ & $\mathbf{1 9 9 5}$ & $\mathbf{1 9 9 4}$ & $\mathbf{1 9 9 3}$ & $\mathbf{1 9 9 2}$ & $\mathbf{1 9 9 1}$ & $\mathbf{1 9 9 0}$ & $\mathbf{1 9 8 9}$ & $\mathbf{1 9 8 8}$ \\
\hline Ouvrages & 1202 & 1095 & 993 & 1033 & 974 & 842 & 1038 & 859 & 924 & 760 & 972 \\
\hline
\end{tabular}

Source: Index Translationum, bibliographie mondiale de la traduction.

Tableau 3

Traductions publiées au Québec

\begin{tabular}{lccccccccccc}
\hline Année & 2003 & 2004 & 2005 & 2006 & 2007 & 2008 & 2009 & 2010 & 2011 & 2012 & 2013 \\
\hline $\begin{array}{l}\text { Livres } \\
\text { traduits }\end{array}$ & 1170 & 1339 & 1636 & 1782 & 1555 & 1824 & 1694 & 1298 & 1355 & 1431 & 1133 \\
\hline $\begin{array}{l}\text { Traductions } \\
\text { de l'anglais }\end{array}$ & 959 & 1101 & 1414 & 1527 & 1241 & 1462 & 1375 & 1109 & 1171 & 1274 & 954 \\
\hline
\end{tabular}

Source: $B A N Q$.

TABLEAU 4

\section{Coéditions Québec / étranger}

\begin{tabular}{llllllllllll}
\hline Année & 2003 & 2004 & 2005 & 2006 & 2007 & 2008 & 2009 & 2010 & 2011 & 2012 & 2013 \\
\hline Coéditions & 197 & 224 & 182 & 199 & 183 & 213 & 134 & 104 & 141 & 105 & 147 \\
\hline
\end{tabular}

Source: BANQ. 